\title{
Modelagem de incertezas na análise espaço-temporal dos níveis freáticos em uma bacia hidrográfica
}

\author{
Rodrigo Lilla Manzione ${ }^{(1)}$, Suzana Druck ${ }^{(2)}$, Gilberto Câmara ${ }^{(1)}$ e Antonio Miguel Vieira Monteiro(1)
}

\begin{abstract}
(1) Instituto Nacional de Pesquisas Espaciais, Divisão de Processamento de Imagens, Av. dos Astronautas, 1758, CEP $12227-010$ São José dos Campos, SP. E-mail: manzione@dpi.inpe.br, gilberto@dpi.inpe.br, miguel,@dpi.inpe.br (2)Embrapa Cerrados, BR 020, Km 18, Rodovia Brasília-Fortaleza, CEP 73310-970 Planaltina, DF. E-mail: suzana@cpac.embrapa.br
\end{abstract}

Resumo - O objetivo deste trabalho foi avaliar a oscilação dos níveis freáticos em uma bacia hidrográfica, no período seco, e modelar a variabilidade espaço-temporal desses níveis por meio de técnicas geoestatísticas multivariadas. A área de estudo foi o alto da Bacia do Rio Jardim, Distrito Federal. O regime hídrico dessa região é marcado pela sazonalidade, com períodos chuvosos (outubro a abril) e secos (maio a setembro) distintos, que determinam o comportamento dos níveis freáticos. Quanto aos fenômenos com indexação espaço-temporal, a geoestatística propõe soluções pelo modelo linear de co-regionalização, permitindo a quantificação e mapeamento das reservas hídricas subterrâneas. Quantificaram-se o volume perdido de água e o risco associado às estimativas pelas medidas de incerteza, criando-se cenários críticos do volume do aquífero explorado na seca. Na seca, observou-se decréscimo de água nesse aqǘfero. O mapa do quantil 10\% indicou áreas favoráveis ao uso da água, em que os níveis freáticos oscilaram menos. O mapa do quantil $90 \%$ indicou áreas com grandes oscilações dos níveis freáticos que contribuem mais para drenagem e recarga do aqüífero.

Termos para indexação: geoestatística, análise espacial, água subterrânea, medidas de incerteza.

\section{Uncertain modeling on spatio-temporal analysis of water table depths in a watershed}

\begin{abstract}
The aim of this work was to evaluate water table depths in a watershed during the dry season as well as modeling space-time variability of water table depths through multivariate geostatistics techniques. The study area was the high portion of Jardim's river watershed, Distrito Federal, Brazil. Water regime of that region is ruled by seasonality, resulting in wet (October until April) and dry (May until September) periods well-defined that control the behavior of the water table. On space-time index phenomena, geostatistics provide solutions through linear co-regionalization model, allowing quantifying and mapping groundwater reserves. The lost water volume was quantified, as well as the associated estimation risk, through uncertain measures, creating critical scenarios of the explored aquifer volume during the dry season. During the dry season, there was a decrease of water in that aquifer. The quantile map 10\% indicated favorable areas for water use, where the phreatic levels oscillated less. The quantile map $90 \%$ indicated areas with great oscillations of the phreatic levels that contribute more to the drainage and recharge of the aquifer.
\end{abstract}

Index terms: geostatistics, spatial analyses, groundwater, uncertain measures.

\section{Introdução}

Mudanças da disponibilidade de recursos hídricos apresentam impactos diretos no meio ambiente e na ocupação do solo pela agricultura. O volume explorável de um aqüífero é uma variável de decisão a ser determinada como parte de um plano de gestão do sistema de águas de uma região, uma vez que os recursos hídricos possuem abrangência muitas vezes regional, municipal e até mesmo estadual, e não apenas na bacia hidrográfica (Hoffmann \& Jackson, 2000).
A fragilidade na exploração desse tipo de recurso está na renovação das águas retiradas, ou recarga do aqüífero, que não se faz na mesma proporção da extração. Isso pode resultar em uma exploração de partes das reservas permanentes do aquífero, com riscos de exaustão, quando a captação não é devidamente monitorada. A perda de água e a recarga de um aqüífero são parâmetros que possuem variabilidade no espaço devido às diferenças que ocorrem nos solos quanto à absorção e retenção da água (de classes de solos, textura, infiltração) e no tempo devido aos efeitos 
climáticos (pluviometria, evapotranspiração) e pela própria utilização dessa água.

No Cerrado brasileiro, a água subterrânea tem forte atuação na agricultura, uma vez que essa região caracteriza-se por períodos chuvosos e secos bem definidos. No período seco, as reservas reguladoras do aqüífero são responsáveis por fornecer água para irrigação e o comportamento das reservas, quanto à distribuição do volume de água, está submetido aos efeitos sazonais da chuva. Considerando que, em épocas de seca, ocorre decréscimo de água no aqüífero e recomposição desse volume no período chuvoso, o quanto se perde de água, nesse sistema, e como ele se recupera são questões fundamentais para o manejo da água (Oliveira et al., 2005). Essas reservas são importantes tanto na irrigação como na manutenção da vegetação natural e do próprio sistema hídrico da região (Klink \& Machado, 2005). A qualidade de renovação do volume de água durante o período chuvoso é um aspecto que deve ser analisado. Por meio da modelagem das incertezas associadas às estimativas sobre a quantidade de água explorada, pode-se mensurar o risco a que um sistema está exposto, permitindo aos analistas verificar possíveis situações extremas que possam ocorrer (Goovaerts, 2002).

O objetivo deste trabalho foi analisar como o processo de oscilação dos níveis freáticos ocorre no espaço e no tempo, modelando sua dinâmica. O trabalho utilizou procedimentos geoestatísticos espaço-temporais (Goovaerts \& Sonnet, 1993; Papritz \& Flühler, 1994; Kyriakidis \& Journel, 1999), os quais consideram a estrutura de co-regionalização entre dados mensais dessa oscilação no período de seca no Cerrado, de maio a setembro. Essas técnicas permitem não só estimar o volume do aquífero explorado no período, como também calcular medidas de incerteza para a modelagem do risco (Goovaerts, 1997), criando assim cenários relativos ao planejamento dos recursos hídricos e avaliação de reservas.

\section{Material e Métodos}

\section{Modelagem de múltiplas funções aleatórias}

Um dos grandes desafios na modelagem de sistemas aquíferos subterrâneos é a caracterização dos níveis freáticos no espaço e no tempo. A geoestatística proporciona três abordagens conceituais para solução de problemas com indexação no espaço e no tempo
(Kyriakidis \& Journel, 1999): modelos de funções aleatórias simples, integrando os componentes espaciais e temporais (Christakos \& Raghu, 1996); vetores de funções aleatórias espaciais (Goovaerts \& Sonnet, 1993); e vetores de séries temporais (Rouhani \& Wackernagel, 1990).

Em virtude de o domínio da informação disponível ser mais abundante no espaço, a geoestatística multivariada apresenta procedimentos espaço-temporais que priorizam o campo de maior informação disponível (Goovaerts \& Sonnet, 1993). As hipóteses adotadas foram: 1) em um instante de tempo, a variação dos níveis freáticos comporta-se como a realização de uma variável regionalizada em um campo aleatório, sendo sua média constante e a variância descrita por um componente estocástico; infere-se ainda que os dados apresentem correlação espacial; 2) em instantes de tempo subseqüentes, as realizações do campo aleatório são correlacionadas e modeladas como uma coleção finita de funções aleatórias espaciais correlacionadas temporalmente.

O modelo parte de um conjunto de dados $\left\{\mathrm{z}\left(\mathrm{u}_{\alpha}, \mathrm{t}\right) ; \alpha=1, \ldots\right.$, $n ; t=1, \ldots, T\}$ observados em $n$ pontos $u_{\alpha}$ do espaço e $T$ pontos t do tempo. Cada valor observadoé interpretado como a realização de uma variável aleatória (VA). Cada conjunto de valores em determinado instante de tempo é uma realização particular de uma função aleatória (FA) espaçotemporal $\{\mathrm{Z}(\mathrm{u}, \mathrm{t})$, em que $(\mathrm{u}, \mathrm{t}) \in \mathrm{D} \times \mathrm{T}\}$ e em que o $\mathrm{D}$ é o domínio espacial e T é o domínio temporal, onde essa FA assume valores. Com base nas hipóteses anteriores, modelouse essa função como uma realização finita para cada instante no tempo de T funções aleatórias espaciais, temporalmente correlacionadas (Goovaerts \& Sonnet, 1993; Papritz \& Flühler, 1994), ou seja, para cada instante do tempo existe uma FA representando a variável em estudo e essas funções relacionam-se entre si. Adotou-se a seguinte representação para o processo espaço-temporal $Z(u, t)$ : $\mathrm{Z}(\mathrm{u}, \mathrm{t})=\left[\mathrm{Z}_{\mathrm{t}}(\mathrm{u}), \mathrm{t}=1, \ldots, \mathrm{T}\right]=\left[\mathrm{Z}_{1}(\mathrm{u}), \ldots, \mathrm{Z}_{\mathrm{T}}(\mathrm{u})\right]^{\prime}$ ou seja, considera-se um conjunto de $\mathrm{T}$ funções aleatórias, cada uma representada por um período de tempo $t$, em que $t=1, \ldots, T$. Supondo estacionaridade intrínseca, ou seja, que o processo é estacionário de primeira ordem e que e o variograma possa ser calculado, para cada uma dessas $\mathrm{T}$ funções aleatórias, tem-se que:

$\mathrm{E}\left[\mathrm{Z}_{\mathrm{t}}(\mathrm{u})-\mathrm{Z}_{\mathrm{t}}(\mathrm{u}+\mathrm{h})\right]=0 \mathrm{e}$

$\gamma_{\mathrm{i} i \mathrm{j}}(\mathrm{h})=1 / 2 \mathrm{E}\left[\left\{\mathrm{Z}_{\mathrm{i}}(\mathrm{u})-\mathrm{Z}_{\mathrm{t}_{\mathrm{i}}}(\mathrm{u}+\mathrm{h})\right\}\left\{\mathrm{Z}_{\mathrm{t}_{\mathrm{j}}}(\mathrm{u})-\mathrm{Z}_{\mathrm{t}_{\mathrm{j}}}(\mathrm{u}+\mathrm{h})\right\}\right] \mathrm{t}_{\mathrm{i}} \mathrm{t}_{\mathrm{j}}=1, \ldots, \mathrm{T}(2)$

Isso indica que a correlação entre quaisquer dados, em dois instantes de tempo e separados pela mesma 
defasagem espacial h é escrita como uma matriz variograma $\Gamma(\mathrm{h})$ :

$\Gamma(h)=\left[\begin{array}{ccccc}\gamma_{\mathrm{t}_{1} \mathrm{t}_{1}}(\mathrm{~h}) & \cdots & \gamma_{\mathrm{t}_{\mathrm{i}} \mathrm{t}_{\mathrm{j}}}(\mathrm{h}) & \cdots & \gamma_{\mathrm{t}_{\mathrm{i}} \mathrm{t}_{\mathrm{T}}}(\mathrm{h}) \\ \vdots & \ddots & \vdots & \ddots & \vdots \\ \gamma_{\mathrm{t}_{\mathrm{i}} \mathrm{t}_{1}}(\mathrm{~h}) & \cdots & \gamma_{\mathrm{t}_{\mathrm{i}} \mathrm{t}_{\mathrm{i}}}(\mathrm{h}) & \cdots & \gamma_{\mathrm{t}_{\mathrm{i}} \mathrm{t}_{\mathrm{T}}}(\mathrm{h}) \\ \vdots & \ddots & \vdots & \ddots & \vdots \\ \gamma_{\mathrm{t}_{\mathrm{T}} \mathrm{t}_{\mathrm{l}}}(\mathrm{h}) & \cdots & \gamma_{\mathrm{t}_{\mathrm{T}} \mathrm{t}_{\mathrm{i}}}(\mathrm{h}) & \cdots & \gamma_{\mathrm{t}_{\mathrm{T}} \mathrm{t}_{\mathrm{T}}}(\mathrm{h})\end{array}\right]$

Os valores da matriz variograma são estimados por meio de:

$\hat{\gamma}_{\mathrm{t}_{\mathrm{i}} \mathrm{t}_{\mathrm{j}}}(\mathrm{h})=\frac{1}{\mathrm{n}} \sum_{\alpha=1}^{\mathrm{n}}\left[\mathrm{z}_{\mathrm{t}_{\mathrm{i}}}\left(\mathrm{u}_{\alpha}\right)-\mathrm{z}_{\mathrm{t}_{\mathrm{i}}}\left(\mathrm{u}_{\alpha}+\mathrm{h}\right)\right]\left[\mathrm{z}_{\mathrm{t}_{\mathrm{j}}}\left(\mathrm{u}_{\alpha}\right)-\mathrm{z}_{\mathrm{t}_{\mathrm{j}}}\left(\mathrm{u}_{\alpha}+\mathrm{h}\right)\right]$

A matriz variograma $\Gamma(h)$ é a matriz de covariância dos incrementos espaciais. Ela descreve a estrutura de correlação em períodos de tempo subseqüentes $\left(\mathrm{t}_{\mathrm{i}}, \mathrm{t}_{\mathrm{j}}\right)=1, \ldots, \mathrm{T}$ (Goovaerts, 1992). Supondo também a não-correlação das VA para grandes distâncias no espaço, ou seja, que o alcance da continuidade espacial é menor que as maiores distâncias do domínio, a matriz variograma tende para uma matriz única de co-variância V:

$\Gamma(\mathrm{h}) \rightarrow \mathrm{V}$ para $\mathrm{hl} \rightarrow \infty$

\section{Análise de co-regionalização}

Um dos aspectos que contribui para melhor compreensão do fenômeno espacial é analisar a sua distribuição em diferentes escalas. Neste trabalho, o termo escala é utilizado de forma intuitiva, sem relação imediata com escalas cartográficas. Microescala são os erros aleatórios, não capturados pela densidade amostral adotada (efeito pepita). Mesoescala são as variações regionais, de menor alcance, e macroescala, as variações globais em alcances maiores. A ocorrência do fenômeno espacial pode se dar em diferentes escalas. Essas diferentes escalas, que podem ser modeladas, referem-se somente ao domínio espacial (geográfico) e as matrizes de variância e co-variância descrevem a estrutura de correlação associada a cada distância (passo) h modelada.

Pelo modelo linear de co-regionalização (MLC), descrito em Goovaerts (1997), supõe-se que a distribuição espacial de uma variável é o resultado da interação de diferentes processos, atuando independentemente em diferentes escalas espaciais. Essas escalas podem ser modeladas pela matriz variograma, usando um modelo aninhado composto por $\mathrm{S}$ funções variogramas. Todos os $\mathrm{T}(\mathrm{T}+1) / 2$ variogramas diretos e cruzados são modelados por uma combinação linear dos variogramas padronizados para um mesmo alcance espacial. Nos casos em que a estrutura $\Gamma(\mathrm{h})$ apresenta dependência da escala espacial, a matriz $\Gamma(\mathrm{h})$ pode ser decomposta em funções de variogramas básicos $\mathrm{g}^{\mathrm{s}}(\mathrm{h})$, em que $\mathrm{s}=1, \ldots, \mathrm{S}$ representa o número de escalas espaciais consideradas (Castrignanò et al., 2000):

$\Gamma(\mathrm{h})=\sum_{\mathrm{s}=1}^{\mathrm{s}} \mathrm{B}^{\mathrm{s}} \mathrm{g}^{\mathrm{s}}(\mathrm{h})$

sendo $\mathrm{B}^{\mathrm{s}}=\left[\mathrm{b}_{\mathrm{t}_{\mathrm{i}} \mathrm{j}}^{\mathrm{s}}\right]$ a matriz $\mathrm{T} \times \mathrm{T}$ positiva semi-definida, chamada de matriz de co-regionalização. Essa matriz fornece descrição mais precisa da estrutura de correlação $\Gamma(\mathrm{h})$ em diversas escalas espaciais, cujos coeficientes $b_{t_{i} t_{j}}^{s}$ expressam a relativa importância da função de variograma básica $\mathrm{g}^{\mathrm{s}}(\mathrm{h})$ em $\Gamma(\mathrm{h})$. Sob a hipótese de estacionariedade de segunda ordem, os variogramas básicos $\mathrm{g}^{\mathrm{s}}(\mathrm{h})$ tendem à unidade quando $\mathrm{lhl}$ tende a infinito, ou seja, $\mathrm{g}^{\mathrm{s}}(\mathrm{h}) \rightarrow 1$ quando $\mathrm{hh} \rightarrow \infty$, e a matriz de variância e co-variância, V, é igual à soma das matrizes de co-regionalização:

$\mathrm{V}=\sum_{\mathrm{s}=1}^{\mathrm{S}} \mathrm{B}^{\mathrm{s}}$

Quando as correlações mudam conforme a escala espacial, as matrizes de co-regionalização $\mathrm{B}^{\mathrm{s}}$ descrevem com maior precisão correlações do que as matrizes variogramas $\Gamma(h)$, já que essas medem apenas a contribuição de cada variável à função variograma (Goovaerts, 1992).

A utilização do modelo de co-regionalização linear implica em considerar que cada $F A Z_{t}(u)$ pode ser decomposta em um conjunto FA não correlacionadas $\left\{\mathrm{Y}_{\mathrm{v}}^{\mathrm{s}}(\mathrm{u}), \mathrm{v}=1, \ldots, \mathrm{T} ; \mathrm{s}=1, \ldots ., \mathrm{S}\right\}$, com coeficientes de transformação $a_{t_{v}}^{s}$ :

$\mathrm{Z}_{\mathrm{t}}(\mathrm{u})=\sum_{\mathrm{s}=1}^{\mathrm{S}} \sum_{\mathrm{v}=1}^{\mathrm{T}} \mathrm{a}_{\mathrm{t}_{\mathrm{v}}}^{\mathrm{s}} \mathrm{Y}_{\mathrm{v}}^{\mathrm{s}}(\mathrm{u})$

em que $Y_{v}^{s}(u)$ são fatores regionalizados do v-ésimo componente na escala espacial s. Para um s fixo, as T FA $Y_{v}^{s}(h)$ têm a mesma função variograma $g^{\mathrm{s}}(\mathrm{h})$. Ao se agrupar os fatores regionalizados com as mesmas funções variogramas $g^{s}(h)$, cada $F A Z_{t}(u)$ pode ser expressa como a soma de FA ortogonais $Z_{t}^{\mathrm{s}}(\mathrm{u})$ :

$\mathrm{Z}_{\mathrm{t}}(\mathrm{u})=\sum_{\mathrm{s}=1}^{\mathrm{S}} \mathrm{Z}_{\mathrm{t}}^{\mathrm{s}}(\mathrm{u})$

Cada componente espacial $Z_{t}^{\mathrm{s}}(\mathrm{u})$ representa o comportamento da FA $Z_{t}(u)$, ou seja, o comportamento 
de $\mathrm{Z}$ no tempo t na escala s. Na decomposição de cada $Z_{\mathrm{t}}(\mathrm{u})$, em diversos componentes $Z_{\mathrm{t}}^{\mathrm{s}}(\mathrm{u})$, pode-se estudar o comportamento temporal da variável $Z$ para diferentes escalas e, para uma dada escala s, a correlação em diferentes períodos é medida pelo coeficiente de correlação estrutural:

$r_{t_{i} t_{j}}^{s}=\frac{b_{t_{i} t_{j}}^{s}}{\sqrt{b_{t_{i} t_{j}}^{s} b_{t_{j} t_{j}}^{s}}}$

Esses coeficientes examinam as correlações entre as variáveis para cada estrutura contida no modelo, revelando comportamentos do fenômeno em escalas definidas, muitas vezes não detectadas por medidas que não tratam o fenômeno em escalas diferentes.

Neste trabalho, a fim de decompor as matrizes $B^{s}$ em matrizes $\mathrm{A}^{\mathrm{s}}$, que correspondem às matrizes compostas pelos coeficientes $a_{t_{v}}^{s}$, descrito na equação 8 , é aplicada a análise de componentes principais, como em Castrignanò et al. (2000). Esses procedimentos têm por objetivo explorar as relações espaço-temporais da variável em estudo nos tempos analisados.

Funções ortogonais são geradas por combinações lineares das funções originais, cujos coeficientes são os autovetores da matriz V. Cada componente principal explica uma porcentagem da variância total que corresponde à razão do seu autovalor e a variância total.

A presença de um modelo de co-regionalização linear, como o apresentado, baseia-se na hipótese de que o processo pode ser explicado por fatores independentes, $\mathrm{Y}_{\mathrm{v}}^{\mathrm{s}}(\mathrm{u})$ como descrito na equação 8. Autovalores calculados a partir da matriz de co-regionalização correspondem a cargas das variáveis nas componentes principais. Com esses valores, constrói-se o gráfico de correlações, assinalando no eixo das abscissas os autovetores da primeira componente para cada variável e, no eixo das ordenadas, os valores da segunda componente para cada variável. Os gráficos mostrando a projeção das variáveis no espaço fatorial são definidos pelas duas componentes principais mais importantes, as quais mostram como se apresenta a estrutura temporal das variáveis em análise.

\section{Local de estudo}

A Bacia do Rio Jardim é uma área representativa de Cerrado, situada na parte Leste do Distrito Federal, entre as latitudes $15^{\circ} 40^{\prime} \mathrm{S} \mathrm{e} 16^{\circ} 02^{\prime} \mathrm{S}$ e as longitudes $47^{\circ} 20^{\prime} \mathrm{W}$ e $47^{\circ} 40^{\prime} \mathrm{W}$, com área de drenagem de 53.796 ha. O clima da área, de acordo com a classificação de Köppen, é do tipo Aw, com temperatura, no mês mais frio, superior a $18^{\circ} \mathrm{C}$, verão chuvoso, inverno seco, e, no mês mais seco, a precipitação é inferior a $60 \mathrm{~mm}$. A estação seca ocorre de maio a setembro, enquanto a estação chuvosa compreende os meses de outubro a abril. Os principais tipos de solo nessa região são (Reatto et al., 2000): Latossolo Vermelho (LV), Latossolo Vermelho-Amarelo (LVA), Cambissolo Háplico (Cx), Plintissolo Háplico (Px), Gleissolo Háplico (Gx) e Neossolo Quartzarênico (RQ) (Figura 1). A ocupação desses solos se dá principalmente por pastagens nativas e cultivadas, campos de milho, soja, feijão, algodão, arroz, e alguns remanescentes de mata nativa, principalmente próximo às nascentes e corpos d'água. Nessa bacia, a irrigação vem sendo intensamente utilizada e sem uma divisão eqüitativa da água disponível, até mesmo com sistemas instalados em áreas com baixa disponibilidade de águas superficiais e em áreas de nascente, o que coloca em risco o sistema de recarga natural dos mananciais existentes (Dolabella, 1996).

A Bacia do Rio Jardim pode ser subdividida em três partes: alto, médio e baixo Jardim, de acordo com a ordenação topológica do rio principal (Dolabella, 1996). A porção do alto Jardim, de 10.121 ha, vem tendo seus níveis freáticos monitorados mensalmente em 41 piezômetros (Lousada, 2005). A distribuição dos piezômetros foi realizada de maneira aleatória de acordo com o mapa de solos, procurando abranger os principais tipos de solo na área (Figura 1). Com base nos níveis freáticos observados, foram gerados variogramas das diferenças entre os níveis freáticos observados em um instante de tempo anterior e os atuais. A defasagem temporal utilizada para cálculo dos acréscimos ou decréscimos de água no solo foi de um mês, no período de maio a setembro de 2004.

\section{Estimativas e medidas de incerteza}

A partir dessa modelagem, estimou-se o valor de $\mathrm{Z}_{\mathrm{t}}\left(\mathrm{u}_{\mathrm{o}}\right)$ para calcular o volume de escoamento natural do aqüífero (VEN) e estimar o risco de perda de água associado ao seu uso. Esse volume faz parte da avaliação das reservas reguladoras do aquífero, que representam a quantidade de água livre armazenada no terreno aquíffero ao curso de uma recarga importante por alimentação natural, ou seja, submetidas ao efeito do ritmo sazonal ou interanual das precipitações (Costa, 2000). Assim, elaboraram-se dois cenários dos valores de água disponível, o primeiro com estratégia de uso da água conservadora e o segundo, com uma estratégia otimista. 
Os valores da VA $\mathrm{Z}_{\mathrm{t}}\left(\mathrm{u}_{\mathrm{o}}\right)$ são estimados por cokrigagem. O objetivo é inferir o valor dessas variáveis no ponto desconhecido do espaço $\mathrm{u}_{\mathrm{o}}$, utilizando a informação disponível nos $\mathrm{T}$ períodos de tempo da variável considerada. Assim, dado um instante de tempo t', $\mathrm{n} \times \mathrm{T}$, a estimativa da função aleatória $\mathrm{Z}(\mathrm{u}, \mathrm{t})$ é dada por:

$\hat{\mathrm{Z}}_{\mathrm{t}}\left(\mathrm{u}_{\mathrm{o}}\right)=\sum_{\mathrm{t}=1}^{\mathrm{T}} \sum_{\alpha=1}^{\mathrm{n}} \lambda_{\alpha \mathrm{t}} \mathrm{z}_{\mathrm{t}}\left(\mathrm{u}_{\alpha}\right)$

Trata-se de uma média ponderada espaço-temporal, em que diferentes pesos $\lambda_{\alpha t}$ são atribuídos em função da dependência espaço-temporal expressa nos variogramas. A partir dessas estimativas $\hat{\mathrm{z}}_{\mathrm{t}}\left(\mathrm{u}_{\alpha}\right), \alpha=1, \ldots, \mathrm{N}$, os mapas de estimativas de volume de escoamento natural do aqüífero $\mathrm{V}_{\mathrm{t}}\left(\mathrm{u}_{\alpha}\right)$ e os desvios-padrão da estimativa nos meses de maio, junho, julho, agosto e setembro são calculados. Nesse caso os estimadores são adaptações da fórmula apresentada em Costa (2000):

$\hat{\mathrm{V}}_{\mathrm{t}}\left(\mathrm{u}_{\alpha}\right)=\mathrm{A} \cdot \mathrm{p}_{\mathrm{ef}} \cdot \mathrm{Z}_{\mathrm{t}}\left(\mathrm{u}_{\alpha}\right), \alpha=1, \ldots \mathrm{N}$

$\hat{\sigma}_{\mathrm{t}}\left(\mathrm{u}_{\alpha}\right)=\mathrm{A} \cdot \mathrm{p}_{\mathrm{er}} \cdot \hat{\sigma}_{\mathrm{Z}_{\mathrm{t}}}\left(\mathrm{u}_{\alpha}\right), \alpha=1, \ldots, \mathrm{N}$

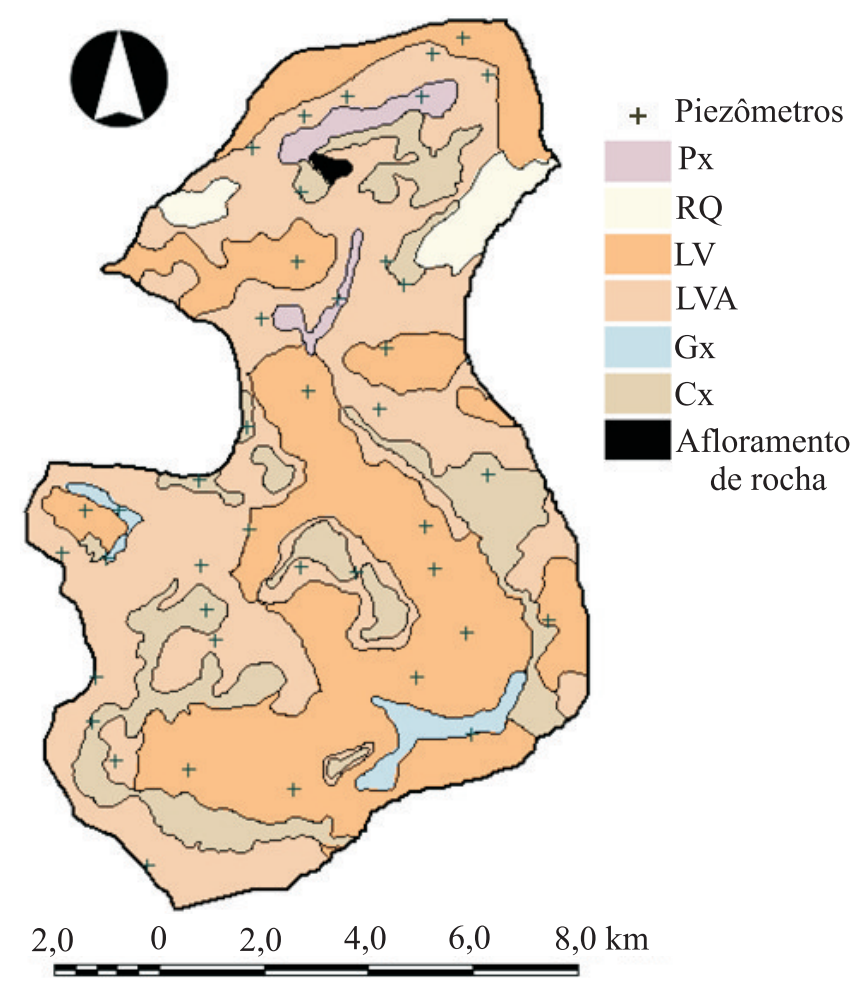

Figura 1. Distribuição dos piezômetros e mapa de solos da Bacia do Rio Jardim, DF. sendo A a área do pixel na grade regular de resolução inferencial e $\mathrm{p}_{\mathrm{ef}}$, a porosidade efetiva para cada tipo de solo considerado na área, (Tabela 1) (Lousada, 2005). Considerando os valores estimados $\hat{\mathrm{V}}_{\mathrm{t}}\left(\mathrm{u}_{\alpha}\right)$ e seu respectivo desvio $\hat{\sigma}_{t}\left(\mathrm{u}_{\alpha}\right)$, pode-se construir uma função de distribuição de frequiências acumuladas para cada ponto. Assumindo-se uma distribuição normal para as oscilações dos níveis freáticos, são calculados os quantis da distribuição de frequiências acumuladas para a geração de cenários que caracterizam o risco associado a essas estimativas pelas medidas de incerteza. Quantis abaixo da mediana são estimativas com alta probabilidade de superestimação, e quantis acima da mediana são estimativas com alta probabilidade de subestimação (Goovaerts, 1997). Os limites críticos adotados foram os quantis $10 \%$ e $90 \%$ :

$\operatorname{Prob}\left[\hat{\mathrm{V}}_{\mathrm{t}}\left(\mathrm{u}_{\alpha}\right)>\mathrm{V}_{\mathrm{q}(0,1)}\right]=0,90$

$\operatorname{Prob}\left[\hat{\mathrm{V}}_{\mathrm{t}}\left(\mathrm{u}_{\alpha}\right)>\mathrm{V}_{\mathrm{q}(0,9)}\right]=0,10$

Esses limites foram considerados adequados nos níveis de incerteza associados à modelagem, e razoáveis no manejo dos recursos hídricos. Dessa forma, foram criados cenários da perda e do ganho de água na área durante o período da seca, proporcionando medidas quantitativas do volume de escoamento natural do aquífero.

\section{Resultados e Discussão}

As distribuições dos dados de acréscimos ou decréscimos de altura dos níveis freáticos apresentaram forte assimetria negativa e altos coeficientes de curtose (Tabela 2). Isso se deve a valores extremos encontrados em função de diferentes respostas do sistema aqüífero apresentadas na área. Enquanto a altura dos níveis freáticos em alguns poços variou apenas alguns centímetros, de um mês a outro, em outros poços, variou alguns metros. Assim, a distribuição dos dados se apresenta com alguns desvios em relação à distribuição normal. O teste de Anderson-Darling (Parkin \&

Tabela 1. Porosidade efetiva mínima (\%) e máxima (\%) estimada para os solos da Bacia do Rio Jardim, DF.

\begin{tabular}{lcc}
\hline Solo & Mínima & Máxima \\
\hline Latossolo Vermelho (LV) & 7 & 9 \\
Latossolo Vermelho-Amarelo (LVA) & 7 & 12 \\
Cambissolo Háplico (Cx) & 3 & 7 \\
Neossolo Quartzarênico (RQ) & 12 & 18 \\
Gleissolo Háplico (Gx) & 7 & 10 \\
Plintossolo Háplico (Px) & 4 & 8 \\
\hline
\end{tabular}


Robinson, 1992) a 0,05\% de probabilidade para verificar a normalidade foi aplicado e não se verificou distribuição normal nos meses em estudo. Com a remoção de alguns outliers oriundos de possíveis erros de medida, as distribuições tiveram sua forma aproximada a uma distribuição normal, e os coeficientes de assimetria e curtose sensivelmente reduzidos.

A estrutura de co-variância direta e cruzada (equação 4) foi estimada pelo modelo de coregionalização linear (Goulard \& Voltz, 1992), apresentado na equação 6 . O modelo apresenta duas estruturas: o efeito pepita $\left(\mathrm{B}^{0}\right)$ ou o componente aleatório de erros em micro-escala e um modelo Gaussiano $\left(\mathrm{B}^{1}\right)$ que explica a variabilidade em escala regional com um alcance comum de 2.130,95 m:

$\begin{aligned} \mathrm{B}^{0} & =\left[\begin{array}{rrrrr}0,0562 & & & & \\ 0,0065 & 0,0490 & & & \\ -0,0138 & -0,0142 & 0,0076 & & \\ -0,0341 & -0,0140 & 0,0001 & 0,1482 & \\ -0,0363 & -0,0285 & 0,0064 & 0,1281 & 0,1169\end{array}\right] \\ \mathrm{B}^{1} & =\left[\begin{array}{rrrrr}0,3196 & & & & \\ 0,3911 & 0,4985 & & & \\ 0,1618 & 0,2289 & 0,2606 & & \\ 0,1725 & 0,2312 & 0,2007 & 0,3354 & \\ 0,1643 & 0,1974 & 0,1517 & 0,1560 & 0,1308\end{array}\right]\end{aligned}$

O modelo foi formulado como $\gamma_{i j}(h)=\mathrm{B}^{0} \mathrm{~g}^{0}(\mathrm{~h})+$ $\mathrm{B}^{1} \mathrm{~g}^{1}(\mathrm{~h})$, sendo $\mathrm{B}^{\mathrm{s}}, \mathrm{s}=0,1$, respectivamente, as matrizes de co-regionalização positivas semi-definidas do efeito pepita e do modelo gaussiano, ajustados aos 15 variogramas diretos e cruzados ajustados (Figura 2).

Tabela 2. Análise estatística descritiva dos dados de acréscimos e decréscimos, em metros, dos níveis freáticos da Bacia do Rio Jardim, DF, de maio a setembro de $2004^{(1)}$.

\begin{tabular}{lcccccccc}
\hline Variável & Média & $\begin{array}{c}\text { Desvio- } \\
\text { padrão }\end{array}$ & Variância & Assimetria & Curtose & $\begin{array}{c}\text { Coeficiente de } \\
\text { variação }\end{array}$ & $\begin{array}{c}\text { Valor P } \\
\text { fistrib. de } \\
\text { freqüência }\end{array}$ \\
\hline Maio & $-0,29$ & 0,70 & 0,48 & $-0,77$ & 4,46 & $-2,39$ & 0,032 & NN \\
Junho & $-0,54$ & 0,67 & 0,45 & $-1,43$ & 5,26 & $-1,24$ & 0,000 & NN \\
Julho & $-0,37$ & 0,50 & 0,25 & $-2,10$ & 7,49 & $-1,35$ & 0,000 & NN \\
Agosto & $-0,55$ & 0,64 & 0,41 & $-1,98$ & 7,00 & $-1,18$ & 0,000 & NN \\
Setembro & $-0,47$ & 0,44 & 0,19 & $-1,10$ & 3,33 & $-0,93$ & 0,000 & NN \\
\hline
\end{tabular}

${ }^{(1)}$ Valor P: teste de Anderson-Darling para normalidade, a 5\% de probabilidade; NN: distribuição não-normal.
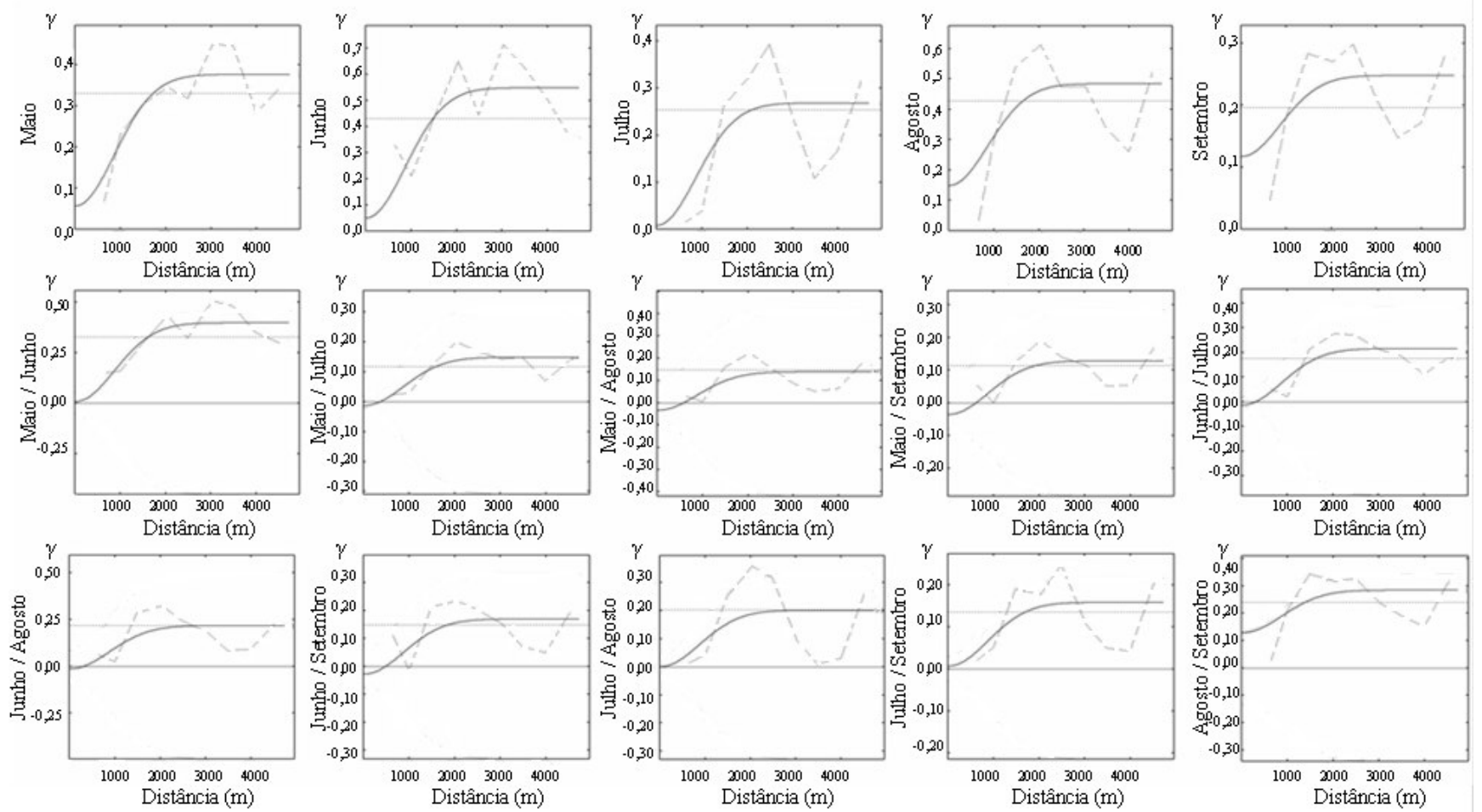

Figura 2. Variogramas experimentais (linha tracejada) e teóricos (linha cheia), diretos e cruzados, ajustados para o período seco analisado. 
As matrizes de co-regionalização apresentadas contêm as contribuições de cada um dos meses em estudo para a estrutura de co-regionalização modelada, expressos na forma de variâncias e co-variâncias.

Nas diagonais, estão as contribuições dos variogramas diretos (variâncias) para cada mês em estudo e fora delas estão as contribuições do variogramas cruzados (co-variâncias entre os meses). Esses resultados são vistos na Figura 2 em forma de variogramas, em que as curvas mostram o modelo proposto ajustado à variância amostral dos dados. As maiores contribuições ao modelo são dadas pelos meses de maio, junho e agosto, que apresentam maiores valores para a estrutura gaussiana (macroescala). Os meses de agosto e setembro apresentam maiores efeitos pepitas, sendo os maiores responsáveis pela componente aleatória do modelo.

A correlação temporal é mais elevada entre os meses mais próximos, conforme os coeficientes de correlação estrutural (equação 12) (Tabela 3). Essas correlações se perdem no tempo devido às modificações nos padrões espaciais de perda e ganho de água na área durante a época seca. Isso pode ser verificado pelo decréscimo nos valores do coeficiente de correlação entre os meses mais distantes. As estimativas de $\hat{\mathrm{z}}_{\mathrm{t}}\left(\mathrm{u}_{\alpha}\right), \alpha=1, \ldots, \mathrm{N}$, ou seja, os acréscimos ou decréscimos em metros dos níveis freáticos na Bacia do Rio Jardim, em cada um desses meses estudados, foram estimadas por cokrigagem (Figura 3).

A análise de componentes principais aplicados $\mathrm{B}^{\mathrm{s}}, \mathrm{s}=0,1$, supondo que sob a hipótese de estacionaridade de segunda ordem, a matriz de variância e co-variância dos atributos $\mathrm{V}$ converge para $\sum_{\mathrm{s}=0}^{\mathrm{S}} \mathrm{B}^{\mathrm{s}}$, revela que a estrutura de autocorrelação em microescala descrita por $\mathrm{B}^{\circ}$ resume $87,96 \%$ da variação do efeito pepita nos dois primeiros

Tabela 3. Coeficientes de correlação estrutural da primeira estrutura do modelo proposto (efeito pepita e modelo gaussiano $\mathrm{a}=2.130,95 \mathrm{~m}$ ) para os meses do período de seca.

\begin{tabular}{|c|c|c|c|c|c|}
\hline Meses & Maio & Junho & Julho & Agosto & Setembro \\
\hline & \multicolumn{5}{|c|}{ Efeito pepita } \\
\hline Maio & 1,00 & - & - & - & - \\
\hline Junho & 0,13 & 1,00 & - & - & - \\
\hline Julho & $-0,66$ & $-0,75$ & 1,00 & - & - \\
\hline Agosto & $-0,37$ & $-0,16$ & 0,03 & 1,00 & - \\
\hline \multirow[t]{2}{*}{ Setembro } & $-0,45$ & $-0,41$ & 0,22 & 0,98 & 1,00 \\
\hline & \multicolumn{5}{|c|}{ Modelo gaussiano } \\
\hline Maio & 1,00 & - & - & - & - \\
\hline Junho & 0,82 & 1,00 & - & - & - \\
\hline Julho & 0,56 & 0,64 & 1,00 & - & - \\
\hline Agosto & 0,53 & 0,57 & 0,70 & 1,00 & - \\
\hline Setembro & 0,80 & 0,76 & 0,79 & 0,80 & 1,00 \\
\hline
\end{tabular}

componentes. Para escalas regionais descritas por $\mathrm{B}^{1}$, as duas primeiras componentes explicam $92,32 \%$ da variação do modelo gaussiano. $\mathrm{O}$ efeito pepita corresponde a $19,66 \%$ da variação do MLC considerando ambas as estruturas modeladas. O modelo Gaussiano corresponde aos $80,34 \%$ da variação do MLC.

O gráfico das correlações (Figura 4) mostra que os meses que apresentaram maior contribuição para o efeito pepita, agosto e setembro, formam agrupamentos (clusters) espaciais. Os meses que tiveram menores contribuições, maio e junho, também formam agrupamentos, o que revela os padrões da correlação temporal presente na modelagem. Na escala regional, o gráfico mostra que os meses que mais contribuem ao modelo gaussiano também se agrupam, no caso maio e junho, assim como os meses de menos contribuição, agosto e setembro.

Agosto e setembro são os meses em que os níveis freáticos começam a demonstrar os efeitos da seca, pois, em meses anteriores, o lençol ainda sofre influência das chuvas que ocorreram em março e abril. A estrutura temporal de variação mais forte vai se perdendo no decorrer dos meses, conforme os coeficientes de correlação estrutural (Tabela 3). Esses resultados ratificam a estrutura de correlação temporal descrita pelos coeficientes de cada escala espacial modelada antes da decomposição da matriz de co-regionalização em fatores independentes, e pelos padrões espaciais encontrados nos mapas estimados por cokrigagem (Figura 3).

Os mapas gerados por cokrigagem para os meses em estudo tiveram seus valores somados para obtenção do volume de escoamento natural do aqüífero em toda área durante o período de seca (Figura 5). Os valores do volume de escoamento natural do aqüífero no período da seca estão indicadas na Tabela 4. As classes de Neossolos Quartazarênicos presentes na área apresentaram sempre grandes decréscimos dos níveis freáticos, devido a sua textura de solo mais grosseira e maior presença de macroporos, que resultam em uma percolação de água mais rápida no perfil, menor retenção de água por capilaridade e secamento mais rápido durante períodos de estiagem prolongados (Resende et al., 2002). Os Latossolos Vermelho-Amarelos apresentaram decréscimos maiores que os Latossolos Vermelhos, uma vez que sua textura é mais arenosa. A água é armazenada por retenção nos poros capilares dos solos e no interior de rochas porosas ou diaclasadas; parte percola devido à saturação, permanecendo no interior das rochas porosas ou entre as fraturas das 
rochas maciças, e parte se perde por evapotranspiração (Lousada, 2005). Entretanto, nas áreas em que são presentes os Latossolos Vermelhos houve tanto grande acréscimo como grande decréscimo no volume de água. Essa variabilidade resulta de diferenças estruturais e texturais dessa classe de solos (Reatto et al., 2000) e também dos processos de ocupação e do manejo a que esses solos vêm sendo submetidos (Dolabella, 1996). O fluxo e a retenção de água no solo dependem da profundidade, textura, estrutura, porosidade e pedoforma, as quais podem se integrar das mais diversas maneiras. Segundo Resende et al. (2002), além da textura, efeitos concorrentes como a mineralogia, porosidade e estrutura são fundamentais para previsão da retenção da água em diferentes classes de solo. A identificação e avaliação desses atributos subsidiam a previsão do comportamento de possíveis impactos no sistema solo-água-vegetação. Os Cambissolos Háplicos apresentaram pequenos decréscimos nos níveis freáticos. Os Plintossolos Háplicos, em geral, apresentaram acréscimos no volume de água, enquanto os Gleissolos Háplicos, em geral, apresentaram decréscimos no volume de água no aqüífero. As áreas que têm acréscimos no volume de água durante o período concentram-se nas cabeceiras da bacia.

Os cenários construídos a partir das estimativas dos quantis, calculados a partir das funções de distribuições acumuladas para cada ponto da grade inferencial, são relevantes para manejo dos recursos hídricos, uma vez que os processos inferenciais podem subestimar ou superestimar valores, e esses erros possuem diferentes conseqüências no planejamento (Goovaerts, 1997).
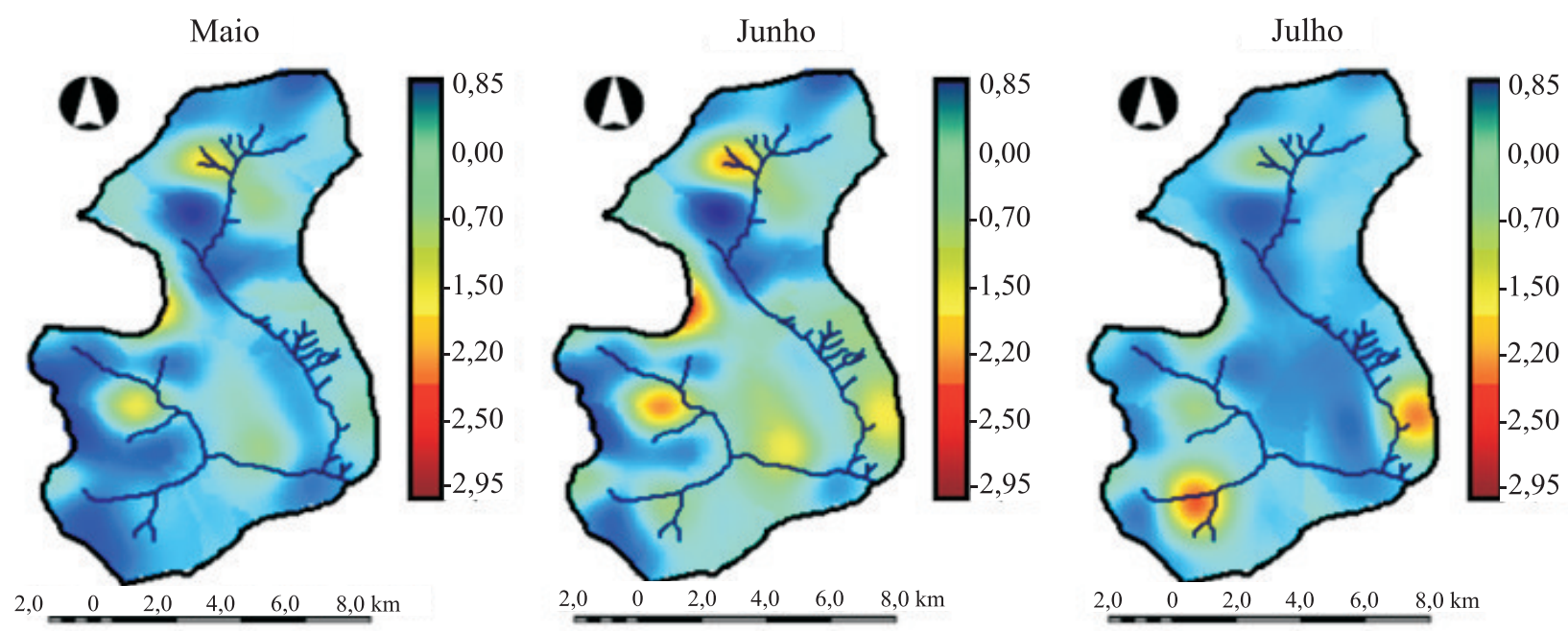

Agosto

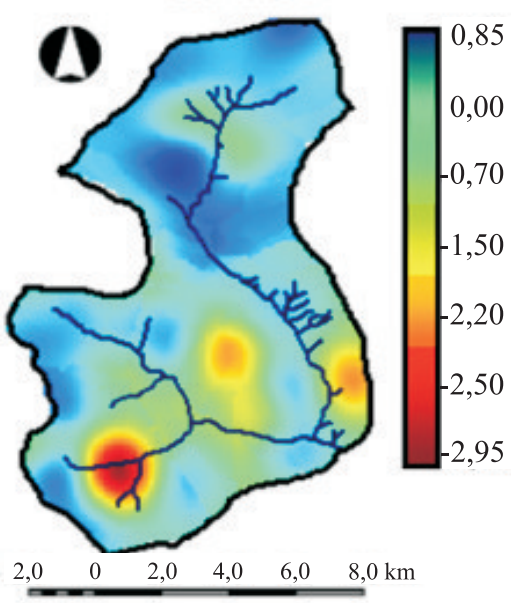

Setembro

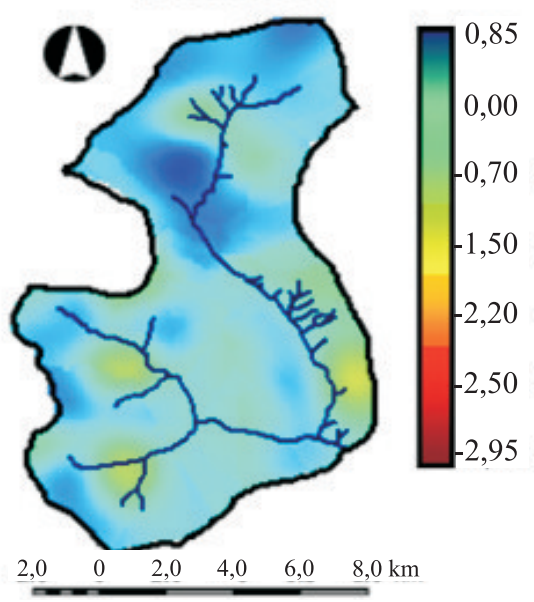

Figura 3. Mapas estimados por cokrigagem para oscilação dos níveis freáticos (em metros) em maio, junho, julho, agosto e setembro na Bacia do Rio Jardim. 
As estimativas dos quantis têm seus resultados exibidos na Tabela 4. Os valores encontrados variam $124 \% \mathrm{em}$ relação aos valores encontrados nas estimativas obtidas por cokrigagem, tanto para mais quanto para menos, uma vez que medem os afastamentos das estimativas em relação à média.

O mapa do quantil de $10 \%$ é um mapa subestimado em relação à média de volume de escoamento natural de água $\left(\mathrm{m}^{3} \mathrm{ha}^{-1}\right)$ calculado a partir dos mapas gerados por cokrigagem para cada mês. Seus valores são sensíveis à superestimação, revelando um cenário pessimista, em que a perda de água no período seco é considerada máxima (Figura 5).

Assim, valores de volume de água apresentados no mapa têm uma probabilidade maior que $90 \%$ de serem

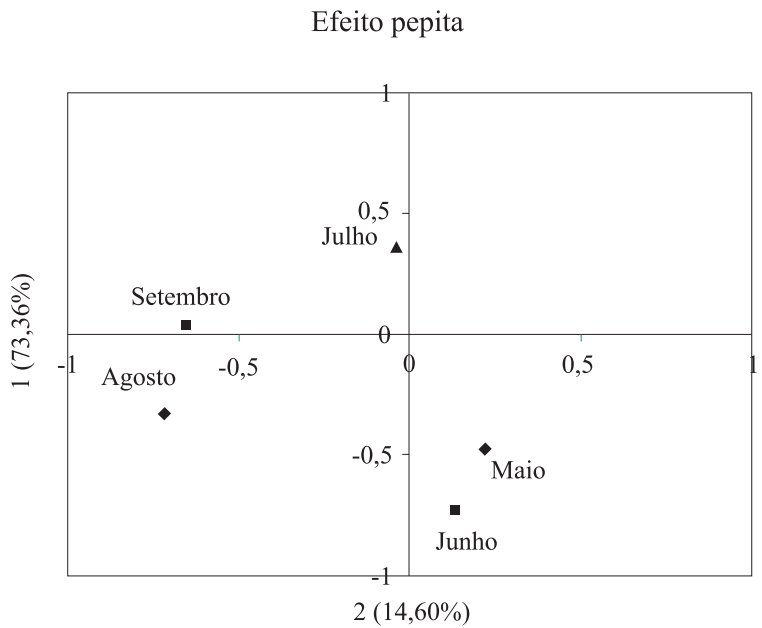

maiores que os apresentados, e apenas de $10 \%$ de serem menores. Esse mapa é um indicativo de áreas onde os decréscimos dos níveis freáticos são menos acentuados e em que a perda de água é efetivamente menor.

Da mesma forma, o mapa do quantil $90 \%$ é um mapa superestimado. Seus valores são sensíveis à subestimação, revelando um cenário otimista em que a perda de água no período seco é considerada mínima (Figura 5). Os valores do mapa têm probabilidade maior que $10 \%$ de ser maiores que os apresentados, e de $90 \%$ de ser menores. As áreas que apresentam as maiores perdas de volume no mapa (áreas com valores mais baixos no mapa) são áreas que têm grande rebaixamento dos níveis freáticos. Esse mapa é um indicativo de áreas em que decréscimos dos níveis freáticos são mais acentuados. O mapa indica regiões

Modelo gaussiano (2130,95 m)

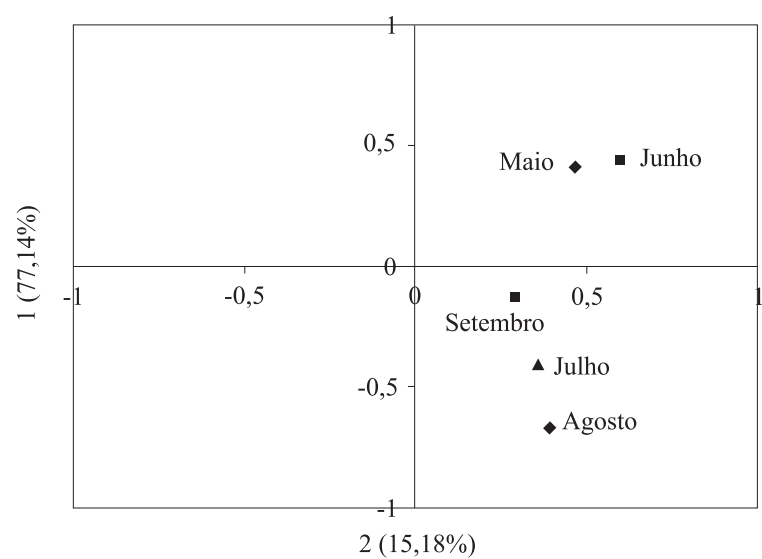

Figura 4. Projeção no espaço definido pelas duas componentes principais mostrando as estruturas modeladas para os meses do período das secas (efeito pepita e modelo gaussiano).

Volume estimado

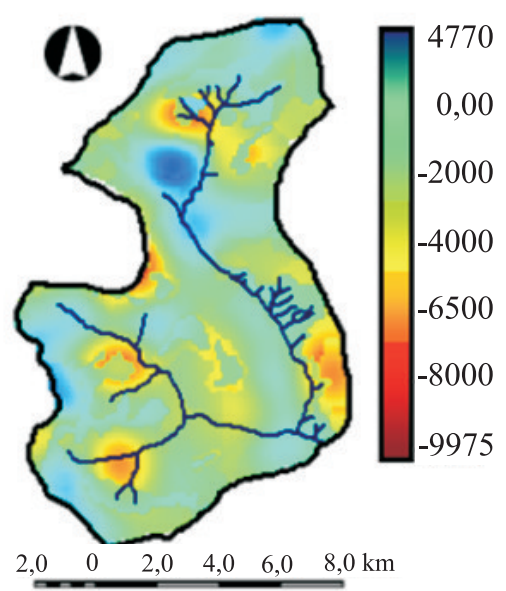

Quantil 0,1

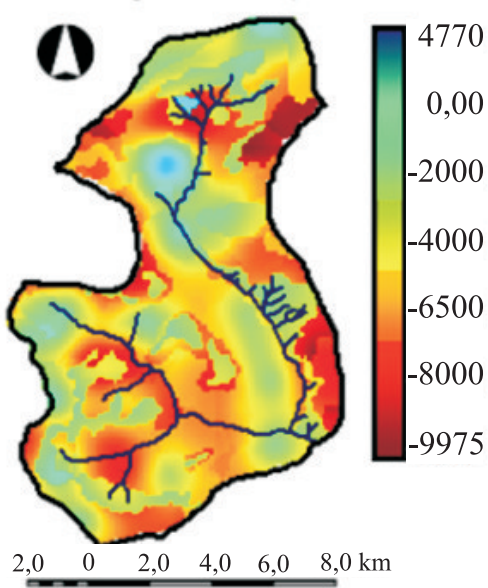

Quantil 0,9

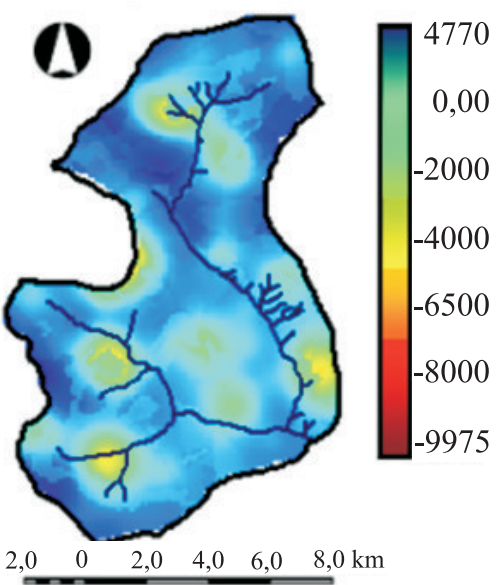

Figura 5. Mapas de volume de escoamento natural do aqüífero na Bacia do Alto Jardim durante o período seco $\left(\mathrm{m}^{3} \mathrm{ha}^{-1}\right)$, estimado por cokrigagem e os respectivos quantis 10 e $90 \%$ calculados. 
Tabela 4. Volume de escoamento natural do aquífero no período da seca na Bacia do Alto Jardim, DF.

\begin{tabular}{lrrc}
\hline & $\begin{array}{c}\text { Total } \\
\left(\mathrm{m}^{3}\right)\end{array}$ & $\begin{array}{c}\text { Hectare } \\
\left(\mathrm{m}^{3} \mathrm{ha}^{-1}\right)\end{array}$ & $\begin{array}{c}\text { Dia } \\
\left(\mathrm{m}^{3} \mathrm{ha}^{-1} \mathrm{dia}^{-1}\right)\end{array}$ \\
\hline Estimado $(\mathrm{X})$ & -22.963 .100 & $-2.069,07$ & $-13,52$ \\
Quantil 10\% & -51.541 .600 & $-4.643,97$ & $-30,35$ \\
Quantil $90 \%$ & 5.603 .300 & 504,87 & 3,29 \\
\hline
\end{tabular}

importantes para a drenagem da área e recarga do sistema aquífero e áreas mais sensíveis aos longos períodos de estiagem.

Áreas com decréscimo nos níveis freáticos possuem alta probabilidade de perder volume de água. Como há grandes variações nas estimativas, avaliações otimistas podem resultar em quebras de produção e erros na localização e instalação de sistemas para captação de água. Considerar a incerteza contribui com a preservação de áreas importantes para recarga do aqüífero. Isto permite uma avaliação realista do impacto da implantação de culturas permanentes ou de grande exigência hídrica.

\section{Conclusões}

1. Uma metodologia que considera a dependência espaçotemporal entre as medidas de altura dos níveis freáticos permite construir mapas de incerteza do volume de escoamento natural de água do aquífero.

2. Mapas de quantil 10\%, média, e quantil $90 \%$, gerados a partir da mesma estrutura de correlação espaço-temporal apresentam grandes diferenças no volume de escoamento natural doaqüífero.

3. O mapa de quantil $10 \%$ indica áreas favoráveis ao uso da água, em que os níveis freáticos oscilam menos; o mapa de quantil $90 \%$ indica áreas com grandes oscilações dos níveis freáticos que contribuem mais para a drenagem e recarga do aqüífero.

\section{Referências}

CASTRIGNANÒ, A.; GIUGLIARINI, L.; RISALITI, R.; MARTINELLI, N. Study of spatial relationships among some soil physico-chemical properties of a field in central Italy using multivariate geostatistics. Geoderma, v.97, p.39-60, 2000.

CHRISTAKOS, G.; RAGHU, R. Dynamic stochastic estimation of physical variables. Mathematical Geology, v.28, p.341-365, 1996.
COSTA, W.D. Uso e gestão de água subterrânea. In: FEITOSA, F.A.C.; MANOEL FILHO, J. (Ed.). Hidrogeologia: conceitos e aplicações. Fortaleza: CPRM, 2000. p.314-367.

DOLABELLA, R.H.C. Caracterização agroambiental e avaliação da demanda e da disponibilidade dos recursos hídricos do Rio JardimDF. 1996. 105p. Dissertação (Mestrado) - Universidade de Brasília, Brasília. GOOVAERTS, P. Factorial kriging analysis: a useful tool for exploring the structure of multivariate spatial soil information. Journal of Soil Science, v.43, p.597-619, 1992.

GOOVAERTS, P. Geostatistical modelling of spatial uncertainty using pfield simulation with conditional probability fields. International Journal of Geographical Information Science, v.16, p.167-178, 2002.

GOOVAERTS, P. Geostatistics for natural resources evaluation. New York: Oxford University Press, 1997. 483p.

GOOVAERTS, P.; SONNET, P. Study of spatial temporal variation of hydrogeochemical variables using factorial kriging analysis. In: SOARES, A. (Ed.). Geostatistics Tróia '92. Dordrecht: Kluwer Academic Publishers, 1993. v.2, p.745-756.

GOULARD, M.; VOLTZ, M. Linear coregionalization model: a toll for estimation and choice of cross-variogram matrix. Mathematical Geology, v.24, p.269-286, 1992.

HOFFMANN, W.A.; JACKSON, R.B. Vegetation-climate feedbacks in the conversion of tropical savanna to grassland. Journal of Climate, v.13, p.1593-1602, 2000.

KLINK, C.A.; MACHADO, R.B. Conservation of the Brazilian Cerrado. Conservation Biology, v.19, p.707-713, 2005.

KYRIAKIDIS, C.P.; JOURNEL, A.G. Geostatistical space-time models: a review. Mathematical Geology, v.31, p.651-684, 1999.

LOUSADA, E.O. Estudos hidrológicos e isotópicos no Distrito Federal: modelos conceituais de fluxo. 2005. 127p. Tese (Doutorado) - Universidade de Brasília, Brasília.

OLIVEIRA, R.S.; BEZERRA, L.; DAVIDSON, E.A.; PINTO, F.; KLINK, C.A.; NEPSTAD, D.C.; MOREIRA, A. Deep root function in soil water dynamics in cerrado savannas of central Brazil. Functional Ecology, v.19, p.574-581, 2005.

PAPRITZ, A.; FLÜHLER, H. Temporal change of spatially autocorrelated soil properties: optimal estimation by cokriging. Geoderma, v.62, p.29-43, 1994.

PARKIN, T.B.; ROBINSON, J.A. Analysis of lognormal data. In: STEWART, B.A. (Ed.). Advances in soil science. New York: Springer-Verlag, 1992. v.20, p.191-235.

REATTO, A.; CORREIA, J.R.; SPERA, S.T.; CHAGAS, C. da S.; MARTINS, E. de S.; ANDAHUR, J.P.; GODOY, M.J.S.; ASSAD, M.L.C.L. Levantamento semidetalhado dos solos da Bacia do Rio Jardim, DF escala 1:50.000. Planaltina: Embrapa Cerrados, 2000. 1 CD-ROM. (Embrapa Cerrados. Boletim de Pesquisa, 18).

RESENDE, M.; CURI, N.; REZENDE, S.B.; CORRÊA, G.F. Pedologia: base para distinção de ambientes. 4.ed. Viçosa: Neput, 2002. 339p.

ROUHANI, S.; WACKERNAGEL, H. Multivariate geostatistical approach to space-time data analysis. Water Resources Research, v.26, p.585-591, 1990.

Recebido em 8 de novembro de 2005 e aprovado em 5 de dezembro de 2006 